\title{
An exactly solvable spherical mean-field plus extended monopole pairing model
}

\author{
Feng Pan, ${ }^{1,2}$ Xiaoxue Ding, ${ }^{1}$ Kristina D. Launey, ${ }^{2} \mathrm{Hui} \mathrm{Li},{ }^{1} \mathrm{Xinxin} \mathrm{Xu},{ }^{1}$ and J. P. Draayer ${ }^{2}$ \\ ${ }^{1}$ Department of Physics, Liaoning Normal University, Dalian 116029, China \\ ${ }^{2}$ Department of Physics and Astronomy, Louisiana State University, Baton Rouge, LA 70803-4001, USA
}

\begin{abstract}
An extended pairing Hamiltonian that describes pairing interactions among monopole nucleon pairs up to an infinite order in a spherical mean field, such as the spherical shell model, is proposed based on the local $\tilde{E}_{2}$ algebraic structure, which includes the extended pairing interaction within a deformed mean-field theory [F. Pan, V. G. Gueorguiev, and J. P. Draayer, Phys. Rev. Lett. 92, $112503(2004)]$ as a special case. The advantage of the model lies in the fact that numerical solutions of the model can be obtained more easily and with less computational time than the solutions to the standard pairing model. Thus, open-shell large-scale calculations within the model become feasible. As an example of the application, pairing contribution to the binding energy of ${ }^{12-28} \mathrm{O}$ is estimated in the present model with neutron pairs allowed to occupy a no-core shell model space of $11 j$-orbits up to the fifth major harmonic oscillator shell including excitations up to $14 \hbar \omega$ for ${ }^{12} \mathrm{O}$ and up to $40 \hbar \omega$ for ${ }^{28} \mathrm{O}$. The results for ${ }^{12} \mathrm{O}$ are also compared and found to be in agreement with those of $a b$ initio calculations. It is shown that the pairing energy per particle in ${ }^{12-28} \mathrm{O}$ ranges from $0.4-$ $1.8 \mathrm{MeV} / A$ with the strongest one observed for a small number of pairs.
\end{abstract}

PACS numbers: 21.60.Fw, 03.65.Fd, 02.20.Qs, 02.30.Ik

\section{INTRODUCTION}

It is well known that the monopole pairing interaction is one of the important residual interactions in a nuclear mean-field theory [1], and is the key to elucidate ground state and low-energy spectroscopic properties of nuclei, such as binding energies, odd-even effects, single-particle occupancies, excitation spectra, and moments of inertia, etc. [1-3]. While the Bardeen-Cooper-Schrieffer (BCS) and the more refined Hartree-Fock-Bogolyubov (HFB) approximations provide a simple and clear demonstration of the role of pairing correlations in nuclei $[2,4,5]$, tremendous efforts have been made in finding accurate solutions to the problem [6-11] to overcome serious drawbacks in the BCS and the HFB, such as spurious states, nonorthogonal solutions, etc., resulting from particle number-nonconservation effects in these approximations [9, 11-13]. It is known that either spherical or deformed mean-field plus the standard pairing interaction can be solved exactly by using the Gaudin-Richardson method [14-16]. It has been shown that the set of Gaudin-Richardson equations can be solved more easily by using the extended Heine-Stieltjes polynomial approach $[17,18]$. However, numerical work in finding roots of the Gaudin-Richardson equations increases with increasing the number of orbits and the number of valence nucleon pairs, which limits the application of the theory within a shell model subspace. On the other hand, the deformed mean-field plus the extended pairing model can be solved more easily than the standard pairing model, especially when both the number of valence nucleon pairs and the number of single-particle orbits are large [19]. It has been proven [20] that the extended pairing model is equivalent to the standard pairing model at a first-step approximation, in which only the lowest energy eigenstate of the standard pure pairing interaction part is considered for solutions of the standard pairing model. The low-lying states obtained in the extended pairing model thus display pair structures similar to the ones of the standard pairing model.

However, the extended pairing interaction proposed in [19] augments a deformed mean-field theory, which can not be used directly to describe pairing interactions among valence nucleon monopole pairs in spherical mean-field theories, in which the total angular momentum of the system is always conserved. The purpose of this work is to establish an extended pairing Hamiltonian to describe pairing interactions among valence nucleon monopole pairs in a spherical mean field similar to the deformed case [19], with which large-scale calculations within the model become feasible.

\section{THE EXTENDED MONOPOLE PAIRING MODEL}

Similar to the extended pairing model for deformed nuclei [19], the Hamiltonian of a spherical mean-field plus the extended monopole pairing model may be written as

$$
\hat{H}=\sum_{j} \epsilon_{j} N_{j}-\sum_{j} G_{j} S_{j}^{\dagger} S_{j}+\hat{H}_{P}
$$


where $\left\{\epsilon_{j}\right\}$ is a set of single-particle energies generated from any spherical mean-field theory, such as those of the spherical harmonic oscillator (HO) shell model, $N_{j}=\sum_{m} a_{j m}^{\dagger} a_{j m}$, in which $a_{j m}^{\dagger}\left(a_{j m}\right)$ is the creation (annihilation) operator for a nucleon with angular momentum quantum number $j$ and that of its projection $m, G_{j}$ is the pairing interaction strength in the $j$-orbit, $S_{j}^{\dagger}=\sum_{m>0}(-1)^{j-m} a_{j m}^{\dagger} a_{j-m}^{\dagger}$ is the monopole pair creation operator in the $j$-orbit,

$$
\hat{H}_{P}=-G \sum_{k=1}^{\infty} \sum_{j_{1} \leq \cdots \leq j_{k}} \tilde{S}_{j_{1}}^{\dagger} \cdots \tilde{S}_{j_{k}}^{\dagger} \sum_{j_{1}^{\prime} \leq \cdots \leq j_{k}^{\prime}} \tilde{S}_{j_{1}^{\prime}} \cdots \tilde{S}_{j_{k}^{\prime}}
$$

in which $G$ is an overall pairing interaction strength for pairing interactions among different orbits, and two groups of orbit indices $\left\{j_{1}, \cdots, j_{k}\right\}$ and $\left\{j_{1}^{\prime}, \cdots, j_{k}^{\prime}\right\}$ in the restricted summation run over all orbits with the restriction that none of $\left\{j_{1}, \cdots, j_{k}\right\}$ equals to any one of $\left\{j_{1}^{\prime}, \cdots, j_{k}^{\prime}\right\}$, but the orbit labels in the same group can be the same, which describes pair hopping from the orbits $\left\{j_{1}^{\prime}, \cdots, j_{k}^{\prime}\right\}$ to the orbits $\left\{j_{1}, \cdots, j_{k}\right\}$ simultaneously. Here,

$$
\tilde{S}_{j}^{\dagger}=\frac{1}{\sqrt{\left(Q_{j}-\hat{S}_{j}^{0}+1\right)\left(Q_{j}+\hat{S}_{j}^{0}\right)}} S_{j}^{\dagger}
$$

in which $Q_{j}=\frac{1}{2}\left(\Omega_{j}-\nu_{j}\right)$ and $\hat{S}_{j}^{0}=\frac{1}{2}\left(N_{j}-\Omega_{j}\right)$ are the quasi-spin and the third component of the quasi-spin operator in the $j$-orbit, respectively, with $\Omega_{j}=j+\frac{1}{2}$ and the seniority number $\nu_{j}$ for the $j$-orbit.

Let $|0\rangle$ be the vacuum state satisfying $a_{j m}|0\rangle=0 \forall j$, which is the lowest-weight state for the quasi-spin $Q_{j}$ when seniority number is zero. For the seniority $\nu_{j} \neq 0$ case, the lowest-weight state for the quasi-spin $Q_{j}$ is denoted as $\left|\nu_{j} \rho_{j}\right\rangle$ satisfying $S_{j}\left|\nu_{j} \rho_{j}\right\rangle=0$, where $\rho_{j}$ stands for the angular momentum quantum number $J_{j}$ and that of its third component $M_{J_{j}}$, and the multiplicity label needed to distinguish different possible ways of $\nu_{j}$ particles coupled to the angular momentum $J_{j}$ under the anti-symmetric irreducible representation $\left\langle 1^{\nu_{j}}\right\rangle$ of $S p(2 j+1) \downarrow O(3)$. Namely, the lowest-weight state with quasi-spin $Q_{j}$, or so-called the pairing vacuum state, is

$$
\left|Q_{j},-Q_{j} ; \nu_{j} \rho_{j}\right\rangle \equiv\left|\nu_{j} \rho_{j}\right\rangle
$$

while

$$
\left|Q_{j},-Q_{j}+n ; \nu_{j} \rho_{j}\right\rangle=\tilde{S}_{j}^{\dagger n}\left|\nu_{j} \rho_{j}\right\rangle
$$

with $n=0,1, \cdots, 2 Q_{j}$, which satisfies

$$
\left(\begin{array}{c}
S_{j}^{\dagger} S_{j} \\
\hat{S}_{j}^{0}
\end{array}\right)\left|Q_{j},-Q_{j}+n ; \nu_{j} \rho_{j}\right\rangle=\left(\begin{array}{c}
n\left(2 Q_{j}-n+1\right) \\
-Q_{j}+n
\end{array}\right)\left|Q_{j},-Q_{j}+n ; \nu_{j} \rho_{j}\right\rangle .
$$

The states with $n$ monopole pairs in the $j$-orbit (5) are orthonormal with respect to $n, \nu_{j}$, and $\rho_{j}$ as long as $\left|\nu_{j} \rho_{j}\right\rangle$ are orthonormalized. It will be assumed that this is the case in the following. Hence, the operator $\hat{S}_{j}^{0}$ appearing in (3) is well defined.

A set of operators $\left\{\tilde{S}_{j_{i}}, \tilde{S}_{j_{i}}^{\dagger}, N_{j_{i}}\right\}(i=1,2, \cdots)$ under the tensor product basis constructed from (5) satisfy the commutation relations

$$
\left[N_{j^{\prime}} / 2, \tilde{S}_{j}\right]=-\delta_{j j^{\prime}} \tilde{S}_{j},\left[N_{j^{\prime}} / 2, \tilde{S}_{j}^{\dagger}\right]=\delta_{j j^{\prime}} \tilde{S}_{j}^{\dagger},\left[\tilde{S}_{j^{\prime}}, \tilde{S}_{j}^{\dagger}\right]=\delta_{j j^{\prime}} \delta_{N_{j} 0}
$$

Therefore, $\left\{\tilde{S}_{j}, \tilde{S}_{j}^{\dagger}, N_{j}\right\}$ for given $j$ is isomorphic to the two-dimensional Euclidean algebra $E_{2}$ when $N_{j} \neq 0$, while it becomes the Heisenberg algebra when and only when $N_{j}=0$, which is called the $\tilde{E}_{2}$ algebra.

To diagonalize the Hamiltonian (1) for $N$ monopole pairs over $p$ orbits, we use the simple algebraic Bethe ansatz with

$$
|N, \zeta\rangle=\sum_{n_{1}, \cdots, n_{p}} C_{n_{1}, \cdots, n_{p}}^{(\zeta)}\left|n_{1}, \cdots, n_{p}\right\rangle=\sum_{n_{1}=0}^{2 Q_{j_{1}}} \cdots \sum_{n_{p}=0}^{2 Q_{j_{p}}} \delta_{\sum_{i=1}^{p} n_{i}, N} C_{n_{1}, \cdots, n_{p}}^{(\zeta)}\left|n_{1}, \cdots, n_{p}\right\rangle
$$

where $\left\{\left|n_{1}, \cdots, n_{p}\right\rangle=\tilde{S}_{j_{1}}^{\dagger n_{1}} \cdots \tilde{S}_{j_{p}}^{\dagger n_{p}}\left|\nu_{j_{1}} \rho_{j_{1}}, \cdots, \nu_{j_{p}} \rho_{j_{p}}\right\rangle\right\}$, which are mutually orthonormal, the sum is restricted to $0 \leq n_{i} \leq 2 Q_{j_{i}}$ and $\sum_{i=1}^{p} n_{i}=N$, and $C_{n_{1}, \cdots, n_{p}}^{(\zeta)}$ are the expansion coefficients to be determined. Actually, the 
dimension of the $N$-pair subspace can be calculated by the counting formula [22]

$$
\operatorname{dim}\left(V_{N}\right)=\sum_{n_{1}=0}^{2 Q_{j_{1}}} \cdots \sum_{n_{p}=0}^{2 Q_{j_{p}}} \delta_{\sum_{i=1}^{p} n_{i}, N}
$$

Similar to the procedures used in [19], it can be proven that the expansion coefficients $C_{n_{1}, \cdots, n_{p}}^{(\zeta)}$ can be expressed as

$$
C_{n_{1}, \cdots, n_{p}}^{(\zeta)}=\frac{1}{F\left(n_{1}, \cdots, n_{p}\right)}
$$

where

$$
F\left(n_{1}, \cdots, n_{p}\right)=E^{(\zeta)}-G-\sum_{i=1}^{p} \epsilon_{j_{i}}\left(2 n_{i}+\nu_{j_{i}}\right)+\sum_{i=1}^{p} G_{j_{i}} n_{i}\left(2 Q_{j_{i}}-n_{i}+1\right)
$$

in which $E^{(\zeta)}$ is the $\zeta$-th eigen-energy. To show that (8) and (10) are indeed consistent, one may directly apply the Hamiltonian (1) on the $N$-pair state (8) to establish the eigen-equation $\hat{H}|N, \zeta\rangle=E^{(\zeta)}|N, \zeta\rangle$. Namely, using that the two groups of orbit indices $\left\{j_{1}, \cdots, j_{k}\right\}$ and $\left\{j_{1}^{\prime}, \cdots, j_{k}^{\prime}\right\}$ in the restricted sum of $\hat{H}_{P}$ run over all orbits with the restriction that none of $\left\{j_{1}, \cdots, j_{k}\right\}$ equals to any one of $\left\{j_{1}^{\prime}, \cdots, j_{k}^{\prime}\right\}$ and after simple algebraic manipulation, one can easily find that

$$
\begin{gathered}
-G \sum_{k=1}^{\infty} \sum_{j_{1} \leq \cdots \leq j_{k}} \tilde{S}_{j_{1}}^{\dagger} \cdots \tilde{S}_{j_{k}}^{\dagger} \sum_{j_{1}^{\prime} \leq \cdots \leq j_{k}^{\prime}} \tilde{S}_{j_{1}^{\prime}} \cdots \tilde{S}_{j_{k}^{\prime}}|N, \zeta\rangle= \\
G|N, \zeta\rangle-G \sum_{n_{1}^{\prime}, \cdots, n_{p}^{\prime}} C_{n_{1}^{\prime}, \cdots, n_{p}^{\prime}}^{(\zeta)} \sum_{n_{1}, \cdots, n_{p}}\left|n_{1}, \cdots, n_{p}\right\rangle .
\end{gathered}
$$

Once the expansion coefficient is chosen as that shown in (11), the eigen-equation $\hat{H}|N, \zeta\rangle=E^{(\zeta)}|N, \zeta\rangle$ is fulfilled when and only when

$$
-G \sum_{n_{1}=0}^{2 Q_{j_{1}}} \cdots \sum_{n_{p}=0}^{2 Q_{j_{p}}} \frac{\delta_{\sum_{i=1}^{p} n_{i}, N}}{F\left(n_{1}, \cdots, n_{p}\right)}=1
$$

Solutions of (13) provide the eigenvalues $E^{(\zeta)}$ and the corresponding eigenstates (8) simultaneously.

When $\epsilon_{j}(j=1, \cdots, p)$ are not equal to one another, which is always the case in the nuclear shell model, for given number of pairs $N=\sum_{j=1}^{p} n_{j}$ with $G>0$, let $x_{\mu}=G+2 \sum_{i=1}^{p} \epsilon_{j_{i}} n_{i}-\sum_{i=1}^{p} G_{j_{i}} n_{i}\left(2 Q_{j_{i}}-n_{i}+1\right)$, which is arranged according to its value for the partition $\left[n_{1}, \cdots, n_{p}\right]$ with $x_{1}<x_{2}<x_{3}<\cdots<x_{\operatorname{dim}\left(V_{N}\right)}$; then zeros of the polynomial given by $(13), E^{(\zeta)}$, satisfy either the interlacing condition $x_{1}<E^{(1)}<x_{2}<E^{(2)}<x_{3}<\cdots$ or $-\infty<x_{1}<E^{(1)}<x_{2}<E^{(2)}<x_{3}<\cdots$, which is very much helpful in finding roots of Eq. (13). Actually, the polynomials associated with Eq. (13) are a special case of the Stieltjes polynomials [17]. When $x_{1}, x_{2}, \cdots, x_{\text {dim }}\left(V_{N}\right)$ are real and not equal to one another, the zeros of the polynomials associated with Eq. (13) are either within $\operatorname{dim}\left(V_{N}\right)$ open intervals $\left(-\infty, x_{1}\right),\left(x_{1}, x_{2}\right), \cdots,\left(x_{\operatorname{dim}\left(V_{N}\right)-1}, x_{\operatorname{dim}\left(V_{N}\right)}\right)$ or within $\left(x_{1}, x_{2}\right),\left(x_{2}, x_{3}\right), \cdots,\left(x_{\operatorname{dim}\left(V_{N}\right)},+\infty\right)$. In this case, the binomials $F\left(n_{1}, \cdots, n_{p}\right)$ of variable $E^{(\zeta)}$ yield different denominators in (13). Therefore, (13) results in a polynomial equation with a variable $E^{(\zeta)}$. The degree of the polynomial equals exactly to the dimension dim( $\left.V_{N}\right)$ of the considered Hilbert subspace. There are exactly $\operatorname{dim}\left(V_{N}\right)$ distinct roots $E^{(\zeta)}$ of $(13)$. Hence, the extended monopole pairing Hamiltonian (1) in this case is exactly solvable. Once the poles $x_{1}, x_{2}, \cdots, x_{\operatorname{dim}\left(V_{N}\right)}$ of $(13)$ are known, it is easy to find the roots of (13) with a suitable computer code for one-variable polynomial equations. As will be used in the following, the smallest root corresponding to the ground state energy should be within the interval $\left(-\infty, x_{1}\right)$ or $\left(x_{1}, x_{2}\right)$, which can easily be obtained by using FindRoot in Mathematica. For the examples provided in the next section, the CPU time needed to find the smallest root of the polynomial equation (13) for polynomial orders less than $10^{7}$ is always less than or about a few seconds on a laptop PC by using Mathematica. The CPU time needed to find other roots is also similar. Therefore, a model with a relatively large dimension can easily be solved numerically with CPU time far less than that required in the standard pairing model [18].

It should be noted that the Hamiltonian (1) is equivalent to the extended pairing model reported in [19] for the Nilsson type mean-field cases when $\Omega_{j}=1 \forall j$. It can be observed that the extended monopole pairing Hamiltonian 
(1) in the double degenerate case becomes the Hamiltonian proposed in [19] when $Q_{j}=1 / 2$ and $G_{j}=G \forall j$ though

(1) is expressed in the way different from that given in Eq. (3) of [19]. Actually, there are $\mu$ ! identical terms with $i_{1} \neq i_{2} \neq \cdots \neq i_{\mu}$ in the summation of the last term in Eq. (3) of [19]. It is obvious that Eq. (3) of [19] can be simplified as (1) with the restricted ordering $j_{1} \leq j_{2} \leq \cdots \leq j_{k}$ and $j_{1}^{\prime} \leq j_{2}^{\prime} \leq \cdots \leq j_{k}^{\prime}$ used in the summation of (2). Since $S_{j}^{\dagger}{ }^{2}=0 \forall j$ in this case, the restricted ordering in the summation can effectively be replaced by $j_{1}<j_{2}<\cdots<j_{k}$ and $j_{1}^{\prime}<j_{2}^{\prime}<\cdots<j_{k}^{\prime}$. Thus, Eq. (8) for the even-even seniority zero case considered in [19] can be rewritten as

$$
|N, \zeta\rangle=\sum_{i_{1}<\cdots<i_{N}} C_{i_{1}, \cdots, i_{N}}^{(\zeta)} S_{i_{1}}^{\dagger} \cdots S_{i_{N}}^{\dagger}|0\rangle,
$$

where $|0\rangle$ is the pairing vacuum state, in which only occupied orbits $i_{1}, i_{2}, \cdots, i_{N}$ are involved.

Since $n_{i}$ can only be taken as 0 or 1 in the double degenerate case, we have $n_{i}^{2}=n_{i} \forall i$. Substituting $Q_{j_{i}}=1 / 2$, $G_{j_{i}}=G, \nu_{j_{i}}=0$, and $\sum_{i} n_{i}=N$ into Eq. (11), one gets

$$
F\left(n_{1}, \cdots, n_{p}\right)=E^{(\zeta)}-G-\sum_{i=1}^{p} 2 \epsilon_{j_{i}} n_{i}+G N .
$$

The $N$-pair excitation energy was defined as

$$
E^{(\zeta)}=\frac{2}{x^{(\zeta)}}-G(N-1)
$$

in [19]. Substituting (16) into (15), one obtains

$$
F\left(n_{1}, \cdots, n_{p}\right)=\frac{2}{x^{(\zeta)}}-\sum_{i=1}^{p} 2 \epsilon_{j_{i}} n_{i}
$$

Since $\sum_{n_{1}=0}^{2 Q_{j_{1}}} \cdots \sum_{n_{p}=0}^{2 Q_{j_{p}}} \delta_{\sum_{i=1}^{p} n_{i}, N} \equiv \sum_{i_{1}<i_{2}<\cdots i_{N}}$ in Eq. (13), in which only occupied orbits $i_{1}, i_{2}, \cdots, i_{N}$ with $n_{i_{\mu}}=1$ are counted for $\mu=1,2 \cdots, N$, Eq. (13) can be rewritten as

$$
1+G \sum_{i_{1}<i_{2}<\cdots<i_{N}} \frac{1}{\frac{2}{x^{(\zeta)}}-\sum_{\mu=1}^{N} 2 \epsilon_{i_{\mu}}}=0
$$

which is identical to Eq. (10) shown in [19]. While the expansion coefficient used in [19] is, up to a constant $x^{(\zeta)} / 2$, equivalent to the expression given in (10). Therefore, the extended pairing interaction reported in [19] becomes a special case of (1) proposed in this paper.

\section{COMPARISON OF THE EXTENDED PAIRING WITH THE STANDARD PAIRING}

In the standard pairing Hamiltonian $[15,16]$, the pairing correlations and their effects are mainly driven by the pairing interactions among different orbits accounted by the non-diagonal part of the Hamiltonian, similar to the role performed by $\hat{H}_{P}$ shown in (1). Hence, while the non-diagonal part of the standard-pairing effects is not present in the first two terms of (1), the $\hat{H}_{P}$ effectively accounts for it. Specifically, the ground state of the pure standard pairing Hamiltonian $\hat{H}_{P}^{s}=-G_{s} S^{\dagger} S[15,16]$, where $G_{s}$ is the overall pairing interaction strength, and $S^{\dagger}=\sum_{i=1}^{p} S_{j_{i}}^{\dagger}$, is given by $[20,21]$

$$
\begin{gathered}
\left|Q^{0}, N\right\rangle=\sqrt{\frac{N !\left(2 Q^{0}-N\right) !}{\left(2 Q^{0}\right) !}} S^{\dagger N}\left|\nu_{j_{1}} \rho_{j_{1}}, \cdots, \nu_{j_{p}} \rho_{j_{p}}\right\rangle= \\
\sqrt{\frac{N !\left(2 Q^{0}-N\right) !}{\left(2 Q^{0}\right) !}} \sum_{n_{1}, \cdots, n_{p}}\left(\prod_{i=1}^{p} \frac{\left(2 Q_{j_{i}}\right) !}{\left(2 Q_{j_{i}}-n_{i}\right) ! n_{i} !}\right)^{1 / 2}\left|n_{1}, \cdots, n_{p}\right\rangle,
\end{gathered}
$$

where $Q^{0}=\sum_{i=1}^{p} Q_{j_{i}}$ is the total quasi-spin for the ground state, with the corresponding eigen-energy

$$
E_{P}^{s}=-G_{s} N\left(2 Q^{0}-N+1\right)
$$


Excited energies of the pure standard pairing Hamiltonian can also be derived from the $S U(2)$ quasi-spin coupling $Q_{j_{1}} \otimes \cdots \otimes Q_{j_{p}} \downarrow Q^{t}$ for $t=0,1,2, \cdots$, of which the corresponding excited states are degenerate, with large degeneracy for each $t$ in general. In contrast to the pure standard pairing Hamiltonian, the extended pairing interaction part of (1) is

$$
\hat{H}_{e x t}=-\sum_{j} G_{j} S_{j}^{\dagger} S_{j}-G \sum_{k=1}^{\infty} \sum_{j_{1} \leq \cdots \leq j_{k}} \tilde{S}_{j_{1}}^{\dagger} \cdots \tilde{S}_{j_{k}}^{\dagger} \sum_{j_{1}^{\prime} \leq \cdots \leq j_{k}^{\prime}} \tilde{S}_{j_{1}^{\prime}} \cdots \tilde{S}_{j_{k}^{\prime}}
$$

In order to check whether (21) results in a similar pair structure as the one produced in the standard pairing Hamiltonian, we set $G_{j}=G_{s} \forall j$ according to the standard pairing model, and adjust $G$ so that the ground state energy of (21) becomes the same as that given by (20). The ground state energy of (21) can be calculated from (13) with $\epsilon_{j}=0 \forall j$. As a nontrivial example, we consider up to the fifth shell in the spherical shell model with $11 j$-orbits: $0 s_{1 / 2}, 0 p_{3 / 2}, 0 p_{1 / 2}, 0 d_{5 / 2}, 2 s_{1 / 2}, 0 d_{3 / 2}, 0 f_{7 / 2}, 1 p_{3 / 2}, 0 f_{5 / 2}, 1 p_{1 / 2}$, and $0 g_{9 / 2}$. In the seniority-zero case, chosen for simplicity, for given total number of pairs $N$, when $G$ is adjusted to produce the ground state energy of the standard pairing (20), we calculate the overlap between the ground state of the extended pairing Hamiltonian (21) and that of the standard pairing Hamiltonian (19) defined by the overlap, $\left|\left\langle Q^{0}, N \mid N, \zeta=1\right\rangle\right|$, where $\left|Q^{0}, N\right\rangle$ is given by (19) for the standard pairing, and $|N, \zeta=1\rangle$ is the ground state of the extended pairing obtained from (8) and (13) with $\epsilon_{j}=0 \forall j$. The results are given in Table I. It is shown in Table I that the $G$ value for producing the same ground state energy in the standard pairing model becomes smaller and smaller when the number of pairs increases towards the half-filling case with $N=Q^{0}$, then increases when the number of pairs increases from the half-filling case towards the fully-filled case with $N_{\max }=2 Q^{0}$. The overlaps change with $N$ in a similar way, namely, they decrease with the increasing number of pairs towards the half-filling case, then similarly increase. The smallest overlap of $78 \%$ occurs near or at the half-filled space with $N=12$ or 13 in this case. Since the $G$ value that reproduces the ground-state energy of the standard pairing model for given $N$ is smaller than $G_{s}$ in general, in order to verify whether the overlap is sensitive to $G$, the overlaps for given $N$ are also calculated with $G=G_{s}$, which are shown in Table II. It is obvious that the overlap for any $N$ changes a little in comparison to the corresponding one shown in Table I, thereby showing that ground-state overlaps are insensitive to $G$. Therefore, though the extended pairing (21) is different from the standard pairing, we show that, as far as the ground state is concerned, the pair structures in the two models are similar, at least when $G \leq G_{s}$ and $G_{j}=G_{s} \forall j$ are set.

TABLE I: $G$ values used in the extended pairing (21) with $G_{j}=G_{s} \forall j$ and for the seniority-zero case that reproduce the respective ground-state energy of the standard pairing (20) for a given total number of pairs $N$, together with the corresponding overlaps $\left|\left\langle Q^{0}, N \mid N, \zeta=1\right\rangle\right|$.

\begin{tabular}{cccccccccccccc}
\hline \hline$N$ & 1 & 2 & 3 & 4 & 5 & 6 & 7 & 8 & 9 & 10 & 11 & 12 \\
\hline$G / G_{s}$ & 2.266 & 0.745 & 0.236 & 0.112 & 0.055 & 0.0304 & 0.0187 & 0.0126 & 0.0093 & 0.00743 & 0.00641 & 0.00596 \\
overlap & 0.99 & 0.93 & 0.93 & 0.88 & 0.86 & 0.84 & 0.82 & 0.81 & 0.80 & 0.79 & 0.79 & 0.78 \\
\hline \hline$N$ & 13 & 14 & 15 & 16 & 17 & 18 & 19 & 20 & 21 & 22 & 23 & 24 & 25 \\
\hline$G / G_{s}$ & 0.00596 & 0.0064 & 0.00743 & 0.0093 & 0.0126 & 0.01868 & 0.0304 & 0.0550 & 0.112 & 0.262 & 0.712 & 2.266 & 1.000 \\
overlap & 0.78 & 0.79 & 0.79 & 0.80 & 0.81 & 0.82 & 0.84 & 0.86 & 0.88 & 0.90 & 0.94 & 0.99 & 1.00 \\
\hline \hline
\end{tabular}

TABLE II: The overlap $\left|\left\langle Q^{0}, N \mid N, \zeta=1\right\rangle\right|$ for a given total number of pairs $N$ with $G_{j}=G_{s} \forall j$ and $G=G_{s}$.

\begin{tabular}{|c|c|c|c|c|c|c|c|c|c|c|c|c|c|}
\hline$N$ & 1 & 2 & 3 & 4 & 5 & 6 & 7 & 8 & 9 & 10 & 11 & 12 & \\
\hline Overlap & 0.98 & 0.94 & 0.92 & 0.89 & 0.87 & 0.85 & 0.83 & 0.82 & 0.81 & 0.80 & 0.80 & 0.80 & \\
\hline$N$ & 13 & 14 & 15 & 16 & 17 & 18 & 19 & 20 & 21 & 22 & 23 & 24 & 25 \\
\hline Overlap & 0.80 & 0.80 & 0.80 & 0.81 & 0.82 & 0.83 & 0.85 & 0.87 & 0.89 & 0.91 & 0.94 & 0.97 & 1.0 \\
\hline
\end{tabular}

\section{APPLICATION TO THE GROUND STATE OF ${ }^{12-28}$ O ISOTOPES}

As a nontrivial application, binding energies and even-odd mass differences of ${ }^{12-28} \mathrm{O}$ are calculated by the spherical shell model plus the extended monopole pairing (1). In order to demonstrate the basic features of the model, we 
consider neutron orbits up to the fifth $\mathrm{HO}$ major shell in the spherical no-core shell model with $11 j$-orbits: $0 s_{1 / 2}$, $0 p_{3 / 2}, 0 p_{1 / 2}, 0 d_{5 / 2}, 2 s_{1 / 2}, 0 d_{3 / 2}, 0 f_{7 / 2}, 1 p_{3 / 2}, 0 f_{5 / 2}, 1 p_{1 / 2}$, and $0 g_{9 / 2}$, though the results for the ground state are similar when only a few less or more $j$-orbits are considered. The neutron single-particle energies $\epsilon_{j}$ are produced from the spherical shell model, in which the usual harmonic oscillator potential, the spin-orbit coupling, and the square of the orbital angular momentum correction are adopted, using a constant $V_{0}=-55 \mathrm{MeV}$ and $\mathrm{HO}$ frequency $\hbar \omega_{0}=41.4678 A^{-1 / 3}\left(1 \pm \frac{A-2 Z}{A}\right)^{1 / 3} \mathrm{MeV}$ with "+" for neutrons and "-" for protons to accommodate the Coulomb potential contribution, where $A$ is the mass number of a nucleus and $Z$ is the total number of protons. Typical neutron single-particle energies and their dependence on $A$ for the oxygen isotopes are shown in Fig. 1 with an example for ${ }^{16} \mathrm{O}$. As the parameters used in the shell model have been extensively studied and adjusted to successfully describe single-particle effects in nuclei [23], the single-particle energies derived in this framework and used here for the pairing model are reasonable. In addition, they are very similar to those obtained from empirical two-body interactions or realistic bare nucleon-nucleon $N N$ interactions (e.g., see [24, 25] for selected cases): the difference between $0 p_{1 / 2}$ and $0 p_{3 / 2}$ in ${ }^{15} \mathrm{O}$ and the differences between $0 d_{3 / 2}, 1 s_{1 / 2}$ and $0 d_{5 / 2}$ in ${ }^{17} \mathrm{O}$, as shown in Fig. 1 , agree with the ones for the unperturbed single-particle energies calculated using the CD-Bonn $N N$ interaction in Ref. [24] (with $\hbar \omega=17$ $\mathrm{MeV}$ for ${ }^{15} \mathrm{O}$ and with $\hbar \omega=16 \mathrm{MeV}$ for ${ }^{17} \mathrm{O}$ ), and for heavier oxygen isotopes toward the neutron-drip line, e.g., the $0 d_{3 / 2}-1 s_{1 / 2}$ gap in ${ }^{24} \mathrm{O}$ agrees with that of Ref. [25].

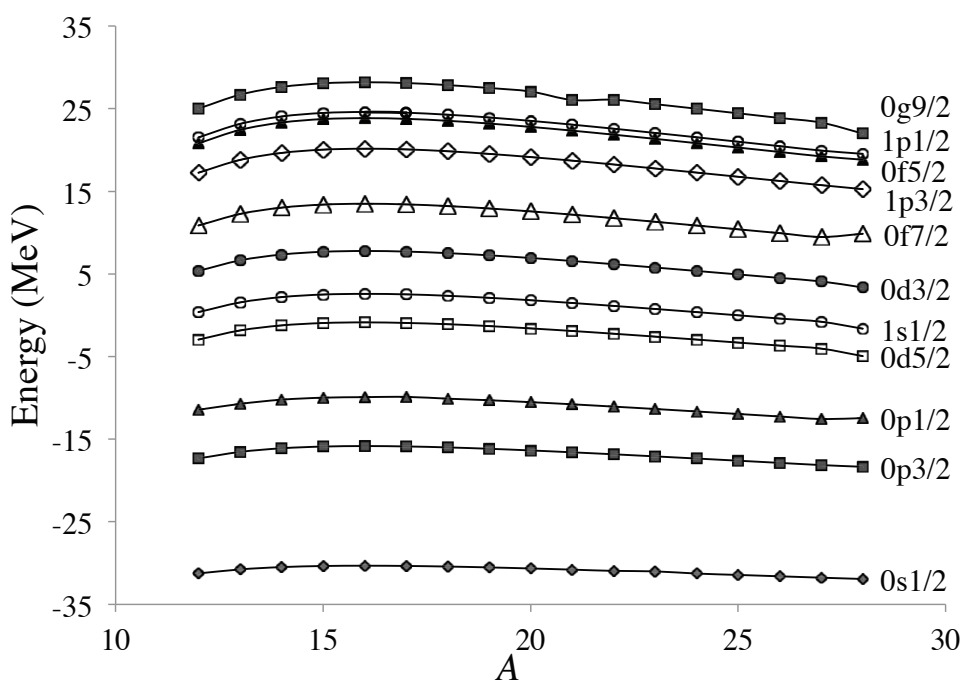

\begin{tabular}{cc}
\hline \hline \multicolumn{2}{c}{${ }^{16} \mathrm{O}$} \\
Single-particle & Neutron energy \\
level & $\epsilon(\mathrm{MeV})$ \\
\hline $0 s_{1 / 2}$ & -30.316 \\
$0 p_{3 / 2}$ & -15.834 \\
$0 p_{1 / 2}$ & -9.910 \\
$0 d_{5 / 2}$ & -0.860 \\
$1 s_{1 / 2}$ & 2.590 \\
$0 d_{3 / 2}$ & 7.781 \\
$0 f_{7 / 2}$ & 13.500 \\
$1 p_{3 / 2}$ & 20.160 \\
$0 f_{5 / 2}$ & 23.860 \\
$1 p_{1 / 2}$ & 24.608 \\
$0 g_{9 / 2}$ & 28.207 \\
\hline \hline
\end{tabular}

FIG. 1: Neutron single-particle energies for oxygen isotopes generated from the spherical shell model as a function of the mass number $A$ (left) and a specific example for ${ }^{16} \mathrm{O}$ (right).

The energy of the ground state of a nucleus with a mass number $A, N$ neutron pairs, and $\nu$ uncoupled neutrons is calculated by the equation

$$
E_{\mathrm{B}}(A)=E_{0}(A)+E_{N, \nu}^{(1)}
$$

where $E_{0}(A)$ accounts for the total energy of the protons plus the average contribution of the neglected interaction, such as the proton-neutron and quadrupole-quadrupole interactions, and $E_{N, \nu}^{(1)}$ is the ground state energy of the neutron part described by the Hamiltonian (1) calculated from (13) with neutron single-particle energies obtained from the shell model. The binding energy obtained from (22) is a reasonable approximation, which assumes that, besides the contribution of the protons and the average of the neglected interaction, the remaining contribution to the binding energy arises mainly from the neutron pairing described within the framework of the shell model with the extended pairing interaction (1). Note that the average contribution of a two-body interaction (often refereed to as "monopole" or "centroid") increases as $A(A-1) / 2$, while that of a one-body interaction increases as $A$, and hence, $E_{0}(A)$ can be approximated by a simple polynomial $v_{0}+v_{1} A+v_{2} A(A-1) / 2$.

As is often done in the standard pairing formalism, the pairing interaction strengths $G_{j}$ used in (1) are assumed, for simplicity, to be the same, namely, $G_{j}=G_{s}=(18 / A)^{1 / 3}(2.1246+2.8197 / 3+2.1845 / 2) / 3=\forall j($ in $\mathrm{MeV})$, 
which is the average value of the $J=0$ two-body diagonal matrix elements of the universal $d s$-shell Hamiltonian of Wildenthal [26]. To find the extended pairing interaction strength $G$ used in (1) for each nucleus, we start with ${ }^{12} \mathrm{O}$, for which we find that for $G=1.18 \mathrm{MeV}$, the ground state energy of the pure extended pairing (21) becomes the same as that given by the pure standard pairing (20) with $G_{s}=1.5861 \mathrm{MeV}$. To further confirm the validity of our choices for the pairing strengths, we study the resulting lowest-energy state for ${ }^{12} \mathrm{O}$ and compare it to the one obtained in $a b$ initio calculations in the framework of the symmetry-adapted no-core shell model (SA-NCSM) [28]. The SA-NCSM calculations use the realistic bare JISP16 nucleon-nucleon interaction for a comparable $\hbar \omega_{0}=17.5$ $\mathrm{MeV}$ and utilize a $N_{\max }=\langle 6\rangle 10$ shell-model space up to $12 \mathrm{HO}$ major shells of dimensionality $6.5 \times 10^{5}$ (the complete model space is included up through $N_{\max }=6$ with symmetry-based selections beyond this, up through $\left.N_{\max }=10\right)$. Indeed, the probability profile for the ${ }^{12} \mathrm{O}$ ground state calculated in the present pairing model closely resembles the one calculated in the $a b$ initio SA-NCSM (Fig. 2a). In contrast, if smaller or larger $G$ values are used (Fig. 2a, inset), the probability profile drastically differs from the one obtained in the ab initio calculations.

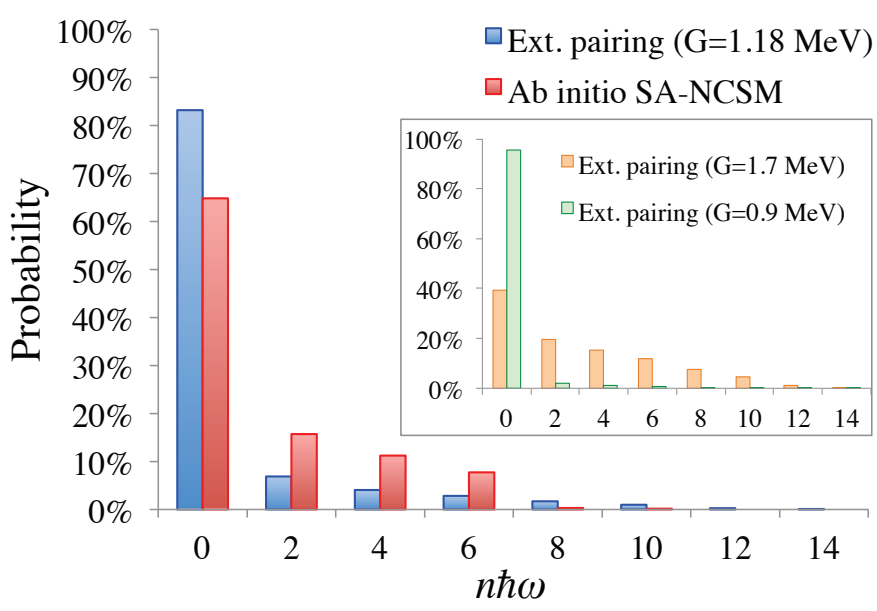

(a)

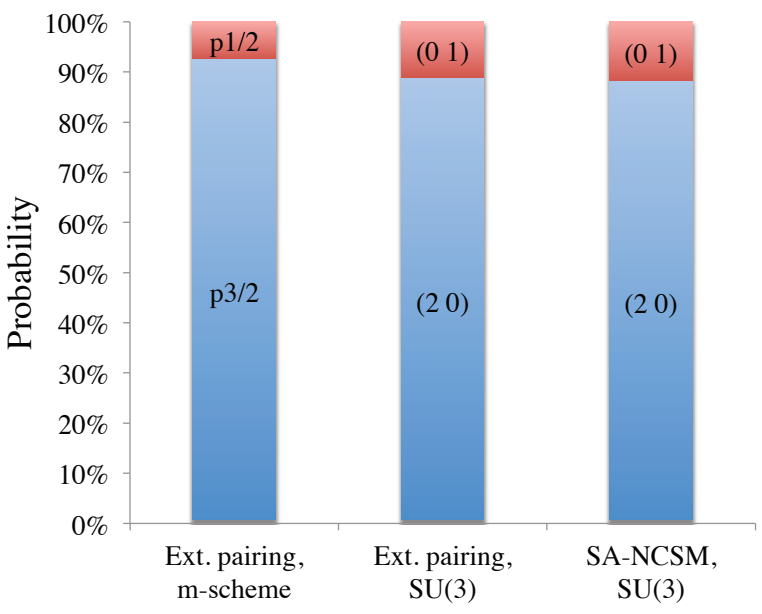

(b)

FIG. 2: (a) Probability distribution of the ${ }^{12} \mathrm{O}$ ground state as a function of the total number of HO excitation quanta (shown along the horizontal axis, $n \hbar \omega=0,2, \ldots, 14 \hbar \omega$ ), obtained in the present extended-pairing model (blue, left bars) and in the $a b$ initio SA-NCSM (red, right bars) with the bare JISP16 nucleon-nucleon interaction in a 12 HO shell-model space and for $\hbar \omega=17.5 \mathrm{MeV}$. For comparison, the probability distributions for a larger $G=1.7 \mathrm{MeV}$ (orange, left bars) and for a smaller $G=0.9 \mathrm{MeV}$ (green, right bars) in the extended pairing model are shown in the inset. (b) Projection of the 0 $\omega \omega$ part of the ${ }^{12} \mathrm{O}$ ground-state wavefunction onto an $m$-scheme basis - a $J=0$ pair in the $0 p_{1 / 2}^{2}$ or $0 p_{3 / 2}^{2}$ configuration - and onto an $\mathrm{SU}(3)$-coupled basis - a $J=0$ pair in the $(\lambda \mu)=(20)$ or $(\lambda \mu)=(01)$ configuration, as compared to the corresponding $a b$ initio SA-NCSM results.

Furthermore, one can study the $\mathrm{SU}(3)$ content of the ${ }^{12} \mathrm{O}$ ground state in the $0 \hbar \omega$ subspace (Fig. 2b). The extended pairing model yields $83 \%$ content in this subspace, with $77 \%$ for a pair in the $0 p_{3 / 2}$ level and with $6 \%$ for a pair in the $0 p_{1 / 2}$ level. This projects onto $\mathrm{SU}(3)$ basis states as following: $74 \%$ for $(\lambda \mu)=(20)$ and $9 \%$ for $(01)$, with $(\lambda \mu)$ being the $\mathrm{SU}(3)$ quantum numbers associated with nuclear deformation. Hence, within the $0 \hbar \omega$ subspace, $(20)$ contributes $88.9 \%$ and $(01)$ contributes $11.1 \%$. Indeed, the ab initio results yield $88.2 \%(20)$ and $11.8 \%(01)$ within the $0 \hbar \omega$ subspace, where the $0 \hbar \omega(20)$ is found to have the largest contribution to the ground state among all possible deformation configurations up through $N_{\max }=10$, followed by $(01)$. This implies that the extended pairing model, in an $N_{\max }=14$ model space, not only detects the two most dominant configurations, but it also closely reproduces their relative contribution. These results suggest that the extended pairing model with the selected $G_{s}$ and $G$ has captured the primary physics that governs the ${ }^{12} \mathrm{O}$ isotope.

Once the $G$ value for ${ }^{12} \mathrm{O}$ is fixed, the $G$ pairing strength in the other isotopes is adjusted to reproduce the experimental even-odd mass difference by using a 4-point difference (corresponding to the third derivative of the energy),

$$
P^{(3)}(A)=\frac{E_{B}(A-2)-3 E_{B}(A-1)+3 E_{B}(A)-E_{B}(A+1)}{4} .
$$

This eliminates any dependence on $A$ and $A^{2}$ and hence on $E_{0}(A)$ of Eq. (22), thereby probing primarily neutron pairing correlations. An excellent agreement of the staggering patterns for the theoretical and experimental $P^{(3)}(A)$ 
with a deviation of only 2 to $70 \mathrm{keV}$ (except a slightly larger one for $A=27$ ), as shown in Fig. 3, allows reasonable values for $G$ to be adopted (given in Table III).

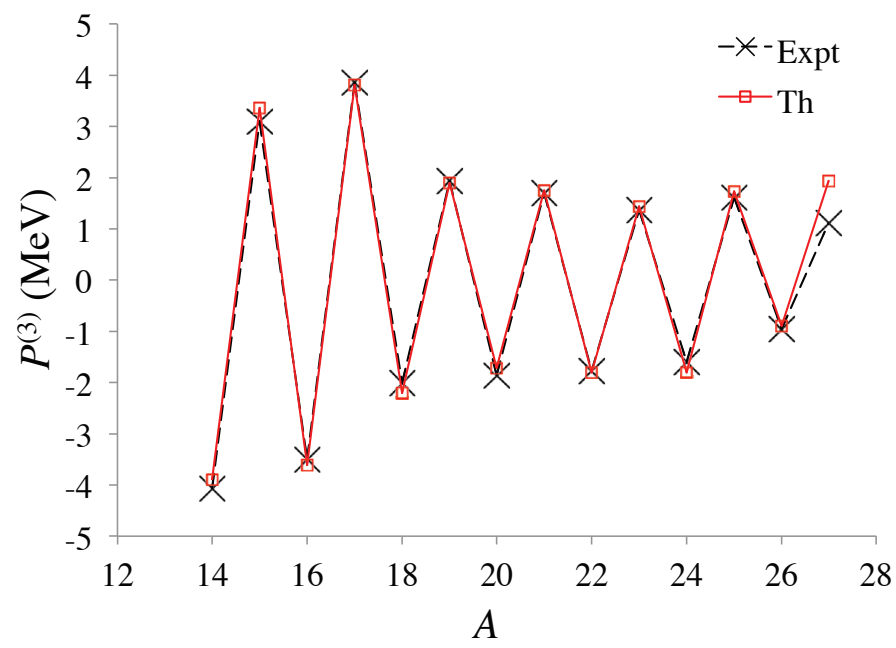

FIG. 3: Four-point even-odd mass difference $P^{(3)}(A)(23)$ obtained from the ground-state energy of the ${ }^{12-28} \mathrm{O}$ isotopes, as measured by experiment ("Expt") [27] and as calculated using Eq. (22) with the model Hamiltonian (1) ("Th").

TABLE III: Number of neutron pairs $N$, pairing interaction strength $G$ (in $\mathrm{MeV}$ ) adjusted to reproduce the experimental 4-point even-odd mass difference for ${ }^{12-28} \mathrm{O}$, theoretical ground-state energy $E_{B}^{\text {th }}$ (in $\mathrm{MeV}$ ) as compared to the experimental values $E_{B}^{\text {expt }}$ (in $\mathrm{MeV}$ ) taken from [27], together with the corresponding dimension of the subspace $V_{N}$.

\begin{tabular}{lccccccccc}
\hline \hline Isotope & ${ }^{12} \mathrm{O}$ & ${ }^{13} \mathrm{O}$ & ${ }^{14} \mathrm{O}$ & ${ }^{15} \mathrm{O}$ & ${ }^{16} \mathrm{O}$ & ${ }^{17} \mathrm{O}$ & ${ }^{18} \mathrm{O}$ & ${ }^{19} \mathrm{O}$ & \\
\hline$N$ & 2 & 2 & 3 & 3 & 4 & 4 & 5 & 5 & \\
$G$ & 1.18 & 1.00 & 0.47 & 0.49 & 0.20 & 0.20 & 0.09 & 0.10 & \\
$E_{B}^{\text {th }}$ & -59.31 & -76.12 & -98.46 & -110.77 & -126.51 & -131.24 & -140.21 & -144.61 & \\
$E_{B}^{\text {expt }}$ & -58.68 & -75.55 & -98.73 & -111.95 & -127.62 & -131.75 & -139.81 & -143.75 & \\
$\operatorname{dim}\left(V_{N}\right)$ & 62 & 61 & 239 & 187 & 708 & 698 & 1716 & 1665 & \\
\hline Isotope & ${ }^{20} \mathrm{O}$ & ${ }^{21} \mathrm{O}$ & ${ }^{22} \mathrm{O}$ & ${ }^{23} \mathrm{O}$ & ${ }^{24} \mathrm{O}$ & ${ }^{25} \mathrm{O}$ & ${ }^{26} \mathrm{O}$ & ${ }^{27} \mathrm{O}$ & ${ }^{28} \mathrm{O}$ \\
\hline$N$ & 6 & 6 & 7 & 7 & 8 & 8 & 9 & 9 & 10 \\
$G$ & 0.040 & 0.055 & 0.020 & 0.040 & 0.010 & 0.028 & 0.008 & 0.021 & 0.0004 \\
$E_{B}^{\text {th }}$ & -152.03 & -155.60 & -162.30 & -164.91 & -169.18 & -167.90 & -167.96 & -165.79 & -169.13 \\
$E_{B}^{\operatorname{expt}}$ & -151.36 & -155.17 & -162.01 & -164.77 & -168.96 & -168.18 & -168.87 & -167.21 & -167.66 \\
$\operatorname{dim}\left(V_{N}\right)$ & 3537 & 3360 & 6361 & 4019 & 10160 & 8161 & 14594 & 11291 & 19019 \\
\hline \hline
\end{tabular}

It can be observed from Table III that the pairing interaction strength $G$ decreases with the increasing number of neutron pairs $N$, and that the $G$ values of odd- $A$ nuclei are systematically larger than the even-even ones with the same number of neutron pairs. A simple fit (of reduced $\chi^{2}=4.7 \times 10^{-4}$ ) to the pairing interaction strength $G$ as a function of the mass number $A$ is preformed with the following expression:

$$
G=3325.7(1+0.32 \nu) \frac{e^{-A / 4}}{A^{2}} \mathrm{MeV}
$$

with $12 \leq A \leq 28$, where $\nu$ is the seniority number with $\nu=0$ for an even-even nucleus, and $\nu=1$ for an odd- $A$ nucleus. As shown in Fig. 4, the pairing strength $G$ decreases with the increasing of $A$ rather smoothly for both even-even and odd- $A$ cases. The power law behavior of (24) is mainly due to the fact that the contribution to the 
binding energy of a nucleus from the pairing interaction among different orbits described by $\hat{H}_{P}$ shown in $(1)$ is proportional to the dimension of the corresponding shell-model subspace $\operatorname{dim}\left(V_{N}\right)$, which increases rather rapidly with the increasing $N$ (see Table III).

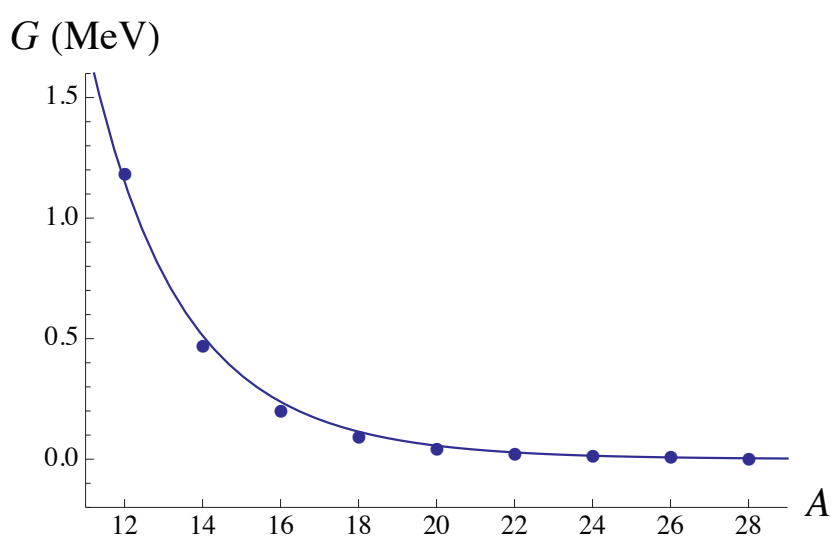

(a)

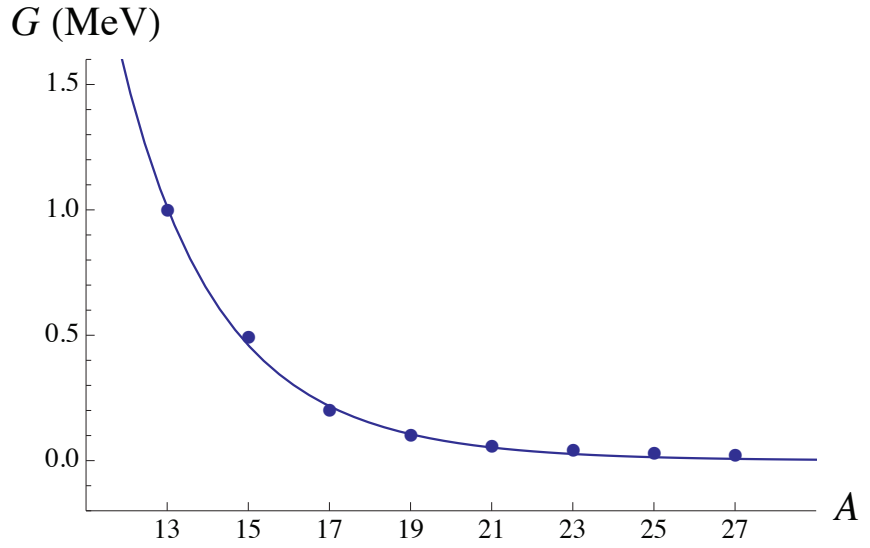

(b)

FIG. 4: Pairing strength $G$ as a function of the mass number $A$ (solid line) obtained by fitting the $G$ values of Table III (dots) for all $\mathrm{A}=12,13, \ldots, 28$ oxygen isotopes using the dependence (24). (a) Even- $A$ and (b) odd- $A$ oxygen isotopes.

Using the $G$ pairing strengths of Table III, $E_{N, \nu}^{(1)}$ of Eq. $(22)$ is then calculated for each $N(\nu=0$ or 1$)$ and hence, the difference $E_{\mathrm{B}}^{\operatorname{expt}}(A)-E_{N, \nu}^{(1)}$ can be used to determine $E_{0}(A)$. The best-fit analysis yields $E_{0}(A)=182.44-15.20 A+$ $0.71\left(\begin{array}{c}A \\ 2\end{array}\right)$ for a reduced $\chi^{2}=0.74$. Once $E_{0}(A)$ is determined, the total ground-state energy is calculated according to Eq. (22), which successfully reproduces the experimental binding energy, shown in Fig. 5a. The 3-point even-odd mass difference (second derivative of energy) is also well reproduced (Fig. 5b). The latter is given by the usual pairing gap expression, $P^{(2)}(A)=E_{B}(A)-E_{B}(A+1)-E_{B}(A-1)$.

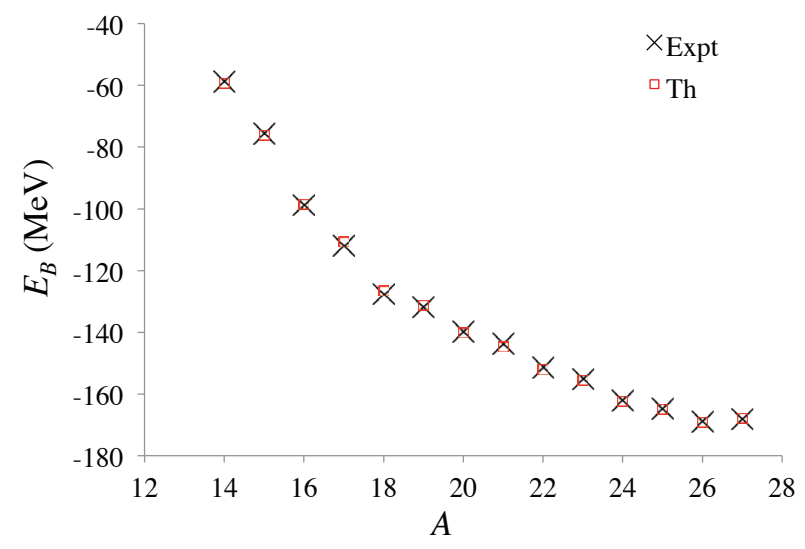

(a)

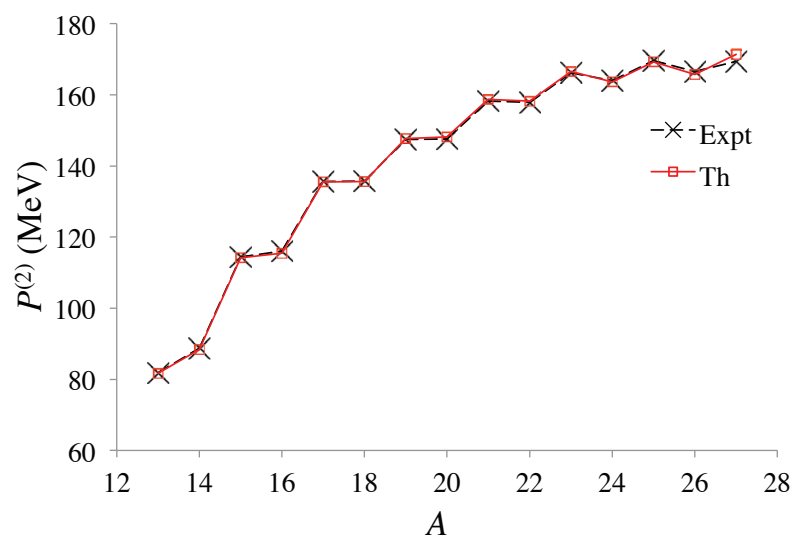

(b)

FIG. 5: Ground-state energy (a) and three-point even-odd mass difference $P^{(2)}(A)$ (b) for ${ }^{12-28} \mathrm{O}$ isotopes. The ground-state energy is calculated using Eq. (22) with the model Hamiltonian $(1)$ and $E_{0}(A)=182.44-15.20 A+0.71\left(\begin{array}{c}A \\ 2\end{array}\right)$ and compared to the experimental data [27].

Fig. 6 shows the pairing energy per particle as a function of the mass number $A$, where the contribution of the pairing energy to the total ground-state energy is calculated by

$$
E_{\text {ext }}=\left\langle N, 1\left|\hat{H}_{\text {ext }}\right| N, 1\right\rangle,
$$

where $\hat{H}_{\text {ext }}$ is given by (21), and $|N, 1\rangle$ is the ground state of the Hamiltonian (1) with $N$ neutron pairs. For the ${ }^{12-28} \mathrm{O}$ isotopes, the pairing energy per particle is on average $-0.8 \mathrm{MeV}$. The largest contribution, about $1.5-1.8$ 
$\mathrm{MeV} / \mathrm{A}$ in magnitude, is observed for $N=2$ and $N=3$ pairs (Fig. 6). Odd- $A$ isotopes with neutrons near to the closed $d s$ shell show a slight decrease from the average, which reflects the fact that the $G$ values for the odd- $A$ isotopes are found to be about 3 times larger than those of the neighboring even- $A$ isotopes with the same number of pairs.

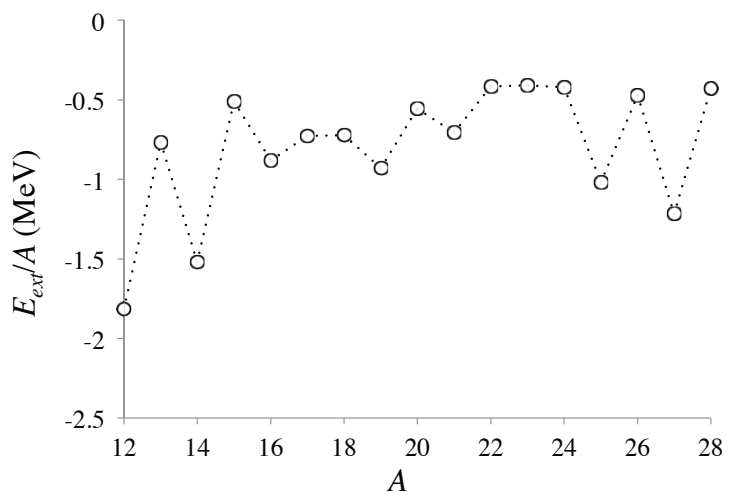

FIG. 6: Pairing energy contribution to the ground-state energy $E_{\text {ext }}$ per particle as a function of the mass number $A$ calculated according to (25).

\section{CONCLUSIONS}

The extended pairing Hamiltonian to describe pairing interactions among nucleon monopole pairs up to infinite order in a spherical mean field, such as the spherical shell model, is proposed based on the local $\tilde{E}_{2}$ algebraic structure, which includes the extended pairing interaction model within a deformed mean-field theory proposed in [19] as a special case. The advantage of the model lies in the fact that numerical solutions of the model can be obtained much more easily with less CPU time than the standard pairing model [17, 18]. Therefore, large-scale calculations with open shell become feasible in the framework of the model. As a demonstration of the power of the model, pairing contributions to the binding energies of ${ }^{12-28} \mathrm{O}$ in the spherical shell model plus the extended pairing model with 11 $j$-orbits up to the fifth major shell are estimated. The results show that the pairing energy per particle in ${ }^{12-28} \mathrm{O}$ ranges from $0.4-1.8 \mathrm{MeV} / A$, and that the strongest pairing occurs for 2-3 pairs. Furthermore, the model successfully reproduces the binding energy of the ${ }^{12-28} \mathrm{O}$ isotopes, together with the even-odd mass difference. In addition, the ground-state wavefunction of ${ }^{12} \mathrm{O}$ is compared to the $a b$ initio SA-NCSM results obtained in the $N_{\max }=\langle 6\rangle 10$ model space using the realistic bare JISP16 nucleon-nucleon interaction and is found to be in a close agreement with the $a b$ initio outcome.

Though only the ground state properties of the model are studied in this work, excited states, such as $0^{+}$pairing excitation states, and other angular momentum nonzero states with the broken-pair consideration, can also be evaluated according to Eq. (13). As shown in Sec. II, the $\tau$-th excited energy $E^{\tau}$ is within the open interval $\left(x_{\tau}, x_{\tau+1}\right)$ or $\left(x_{\tau-1}, x_{\tau}\right)$. The CPU time cost in finding the root within the intervals is the same as that for the ground state. Further analysis on related issues will be our next work.

Once the mean-field plus the extended pairing model is solved, we can also consider other residual interactions, such as the quadrupole-quadrupole interactions among valence particles by using the progressive diagonalization scheme proposed in [20], from which the actual pairing interaction strength can then be properly deduced. This will be part of our future work. The extended pairing interaction among bosons can also be worked out similarly. As shown in our recent work [29], the solvable extended $\mathrm{SU}(1,1)$ pairing interactions among $s$ and $d$ bosons up to an infinite order within the framework of the interacting boson model (IBM) has been used to gain a better description of the E(5) model results in the IBM.

Support from the U.S. National Science Foundation (OCI-0904874), U.S. Department of Energy (DE-SC0005248), the Southeastern Universities Research Association, the China-U.S. Theory Institute for Physics with Exotic Nuclei (CUSTIPEN) (DE-FG02-13ER42025), the National Natural Science Foundation of China (11175078, and 11375080), 
and the LSU-LNNU joint research program (9961) is acknowledged.

[1] P. Ring and P. Schuck, The Nuclear Many-Body Problem (Springer Verleg, Berlin, 1980).

[2] A. Bohr, B. R. Mottelson, and D. Pines, Phys. Rev. 110, 936 (1958); S. T. Belyaev, Mat. Fys. Medd. K. Dan. Vidensk. Selsk. 31, 11 (1959).

[3] M. Hasegawa and S. Tazaki, Phys. Rev. C 47, 188 (1993).

[4] H. C. Pradhan, Y. Nogami, and J. Law, Nucl. Phys. A 201, 357(1973).

[5] H. J. Mang, Phys. Rep. 18, 325 (1975).

[6] G. D. Dans and A. Klein, Phys. Rev. 143, 735 (1966).

[7] A. Covello and E. Salusti, Phys. Rev. 162, 859 (1967).

[8] M. Bishari, I. Unna, and A. Mann, Phys. Rev. C 3, 1715 (1971).

[9] J. Y. Zeng, C. S. Cheng, Nucl. Phys. A 405, 1 (1983); 411, 49 (1984); 414, 253 (1984).

[10] H. Molique, J. Dudek, Phys. Rev. C 56, 1795 (1997).

[11] A. Volya, B. A. Brown, and V. Zelevinsky, Phys. Lett. B 509, 37 (2001).

[12] A. K. Kerman and R. D. Lawson, Phys. Rev. 124, 162 (1961).

[13] V. Zelevinsky and A. Volya, Physics of Atomic Nuclei 66, 1781 (2003).

[14] M. Gaudin, J. Physique 37, 1087 (1976).

[15] R. W. Richardson, Phys. Lett. 3, 277 (1963); 5, 82 (1963); R. W. Richardson and N. Sherman, Nucl. Phys. 52, 221 (1964); 52, 253 (1964).

[16] J. Dukelsky, S. Pittel, and G. Sierra, Rev. Mod. Phys. 76, 643 (2004).

[17] X. Guan, K. D. Launey, M. Xie, L. Bao, F. Pan, J.P. Draayer, Phys. Rev. C 86, 024313 (2012).

[18] X. Guan, K. D. Launey, M. Xie, L. Bao, F. Pan, J.P. Draayer, Comp. Phys. Commun. 185, 2714 (2014).

[19] F. Pan, V. G. Gueorguiev, and J. P. Draayer, Phys. Rev. Lett. 92, 112503 (2004).

[20] F. Pan, M. Xie, X. Guan, L.-R Dai, and J. P. Draayer, Phys. Rev. C 80, 044306 (2009).

[21] G. Racah, Phys. Rev. 62, 438 (1942).

[22] F. Pan, B. Li, Y.-Z. Zhang, and J. P. Draayer, Phys. Rev. C 88, 034305 (2013).

[23] T. Bengtsson and I. Ragnarsson, Nucl. Phys. A 436, 14 (1985).

[24] S. Fujii, R. Okamoto, and K. Suzuki, Phys. Rev. C 69, 034328 (2004).

[25] T. Otsuka, R. Fujimoto, Y. Utsuno, B. A. Brown, M. Honma, and T. Mizusaki, Phys. Rev. Lett. 87, 082502 (2001).

[26] B. H. Wildenthal, Prog. Part. Nucl. Phys. 11, 5 (1984).

[27] M. Wang, G. Audi, A. H. Wapstra, F. G. Kondev, M. MacCormick, X. Xu, and B. Pfeiffer, Chin. Phys. C 36, 1603 (2012).

[28] T. Dytrych, K. D. Launey, J. P. Draayer, P. Maris, J. P. Vary, E. Saule, U. Çatalyürek, M. Sosonkina, D. Langr, and M. A. Caprio, Phys. Rev. Lett. 111, 252501 (2013).

[29] F. Pan, Y. Zhang, H.-C. Xu, L.-R. Dai, and J. P. Draayer, Phys. Rev. C 90, 034305 (2015). 